\title{
Upcoming Meetings Related to Alzheimer's Disease
}

\author{
JANUARY 2016 \\ Conference: \\ Human Amyloid Imaging Conference \\ Date: \\ 13-15 January 2016 \\ Location: Miami, FL, USA \\ URL: \\ http://worldeventsforum.com/hai/ \\ Conference: \\ 14th Annual Mild Cognitive Impairment Symposium/Workshop/Forum \\ Date: \\ 16-17 January 2016 \\ Location: Miami, FL, USA \\ URL: $\quad$ http://www.mcisymposium.org/ \\ Conference: $\quad$ Traumatic Brain Injury: Clinical, Pathological and Translational Mechanisms \\ Date: 24-27 January 2016 \\ Location: Santa Fe, NM, USA \\ URL: \\ http://www.keystonesymposia.org/index.cfm?e=web.Meeting.Program\&meetingid=1424 \\ Conference: \\ Axons: From Cell Biology to Pathology \\ Date: \\ Location: \\ 24-27 January 2016 \\ URL: \\ Santa Fe, NM, USA \\ http://www.keystonesymposia.org/index.cfm?e=web.Meeting.Program\&meetingid=1422 \\ Conference: \\ Neurological Disorders of Intracellular Trafficking \\ Date: \\ Location: \\ 31 January-4 February 2016 \\ URL: \\ Keystone, CO, USA \\ http://www.keystonesymposia.org/index.cfm?e=web.Meeting.Program\&meetingid=1384 \\ MARCH 2016 \\ Conference: \\ Date: \\ Location: \\ URL: \\ 10th Annual Drug Discovery for Neurodegeneration Conference \\ 6-8 March 2016 \\ Miami, FL, USA \\ http://www.alzdiscovery.org/events/conference/10th-annual-drug-discovery-for- \\ for-neurodegeneration-conference \\ Conference: $\quad$ 14th International Athens/Springfield Symposium on Advances in Alzheimer Therapy \\ Date: 9-12 March 2016 \\ Location: Athens, Greece \\ URL: $\quad$ http://www.ad-springfield.com/ \\ Conference: $\quad 2016$ Annual Meeting of the United States \& Canadian Academy of Pathology \\ Date: $\quad 12-18$ March 2016 \\ Location: Seattle, WA, USA \\ URL: $\quad$ http://www.uscap.org/meeting/863333
}




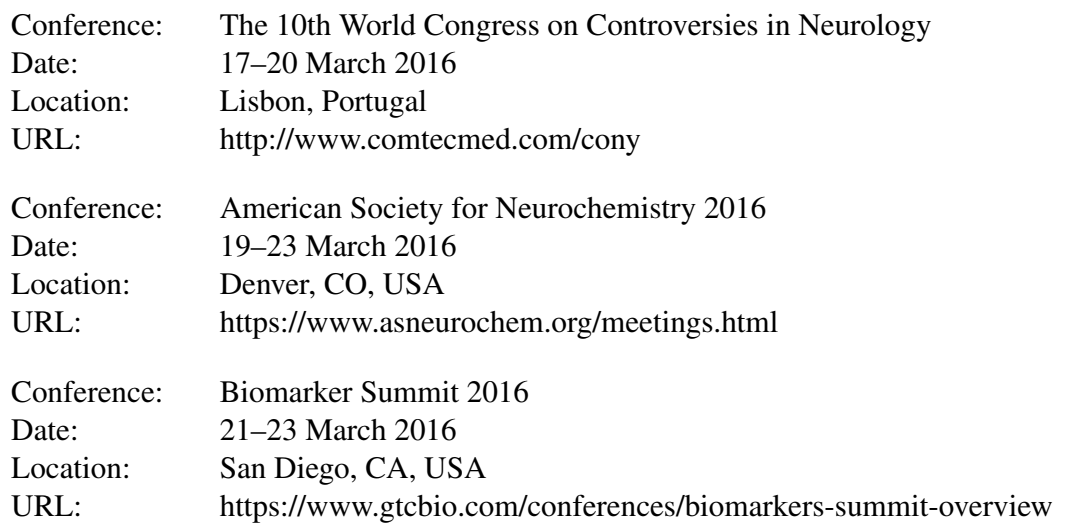

\section{APRIL 2016}

Conference:

Date:

Location:

URL:

Conference:

Date:

Location:

URL:

Conference:

Date:

Location:

URL:

Conference:

Date:

Location:

URL:

Conference:

Date:

Location:

URL:

Conference:

Date:

Location:

URL:

MAY 2016

Conference:

Date:

Location:

URL:

Conference:

Date:

Location:

URL: 18th International Neuroscience Winter Conference

2-6 April 2016

Sölden Austria

http://www.winterneuroscience.org/2016/

13th Annual Congress of the Society for Brain Mapping \& Therapeutics

8-10 April 2016

Miami, FL, USA

http://www.worldbrainmapping.org/general

2016 Zilkha Symposium on Alzheimer Disease \& Related Disorders

10 April 2016

Los Angeles, CA, USA

http://www.keck.usc.edu/events/2016-zilkha-symposium-on-alzheimer-disease-related-disorders/

68th American Academy of Neurology Annual Meeting

15-21 April 2016

Vancouver, BC, Canada

https://www.aan.com/conferences/2016-annual-meeting/

9th International Symposium on Neuroprotection and Neurorepair (ISN\&N)

19-22 April 2016

Leipzig, Germany

http://www.neurorepair-2016.de/

31 st International Conference of Alzheimer's Disease International (ADI)

20-24 April 2016

Budapest, Hungary

http://www.alzint.org/2016

Epigenetic and Metabolic Regulation of Aging and Aging-Related Diseases

1-5 May 2016

Santa Fe, NM, USA

https://www.keystonesymposia.org/index.cfm?e=web.Meeting.Program\&meetingid=1380

6th Annual Traumatic Brain Injury Conference

11-12 May 2016

Washington, DC, USA

http://tbiconference.com/home/ 
Conference:

Date:

Location:

URL:

Conference:

Date:

Location:

URL:

\section{JUNE 2016}

Conference:

Date:

Location:

URL:

Conference:

Date:

Location:

URL:

Conference:

Date:

Location:

URL:

Conference:

Date:

Location:

URL:

\section{JULY 2016}

Conference:

Date:

Location:

URL:

Conference:

Date:

Location:

URL:

\section{AUGUST 2016}

Conference:

Date:

Location:

URL:

\section{SEPTEMBER 2016}

Conference:

Date:

Location:

URL:
2016 Annual Scientific Meeting of the American Geriatrics Society (AGS)

19-21 May 2016

Long Beach, CA, USA

http://www.americangeriatrics.org/annual_meeting/2016_annual_meeting/

European Academy of Neurology Conference 2016

28-31 May 2016

Copenhagen, Denmark

https://www.eaneurology.org/

The 2016 Alzheimer's Disease Congress

7-9 June 2016

London, UK

http://lifescienceevents.com/Alz2016/

Common Mechanisms of Neurodegeneration

12-16 June 2016

Keystone, CO, USA

https://www.keystonesymposia.org/index.cfm?e=web.Meeting.Program\&meetingid=1423

20th International Congress of Parkinson's Disease and Movement Disorders

19-23 June 2016

Berlin, Germany

http://www.mdscongress2016.org/Congress-2016.htm

22nd Annual Meeting of the Organization for Human Brain Mapping

26-30 June 2016

Geneva, Switzerland

http://www.humanbrainmapping.org/i4a/pages/index.cfm?pageID=3662

10th FENS Forum of Neuroscience

2-6 July 2016

Copenhagen, Denmark

http://forum2016.fens.org/

Alzheimer's Association International Conference

22-28 July 2016

Toronto, Canada

https://www.alz.org/aaic/

10th International Conference on Frontotemporal Dementias

31 August - 2 September 2016

Munich, Germany

http://www.icftd2016.de/

41st Congress of The Federation of European Biochemical Societies (FEBS)

3-8 September 2016

Ephesus/Kusadasi, Turkey

http://www.febs2016.org/ 
Conference:

Date:

Location:

URL:

\section{OCTOBER 2016}

Conference:

Date:

Location:

URL:

Conference:

Date:

Location:

URL:

\section{DECEMBER 2016}

Conference:

Date:

Location:

URL:

\section{MARCH 2017}

Conference:

Date:

Location:

URL:

Conference:

Date:

Location:

URL:

APRIL 2017

Conference:

Date:

Location:

URL: 4th World Parkinson Congress (WPC 2016)

20-23 September 2016

Portland, OR, USA

http://www.worldpdcoalition.org/?page=WPC2016

11th International Congress on Non-Motor Dysfunctions in Parkinson's Disease and Related Disorders 6-9 October 2016

Ljubljana, Slovenia

http://nmdpd2016.kenes.com/landing/Pages/default.aspx

VasCog: The International Society of Vascular Behavioural and Cognitive Disorders Conference 12-15 October 2016

Amsterdam, The Netherlands

http://vas-cog.org/welcome+to+vascog/13249/Page.aspx

ICNE 2016: 18th International Conference on Neurology and Epidemiology

12-13 December 2016

Bangkok, Thailand

https://www.waset.org/conference/2016/12/bangkok/ICNE

13th International Conference on Alzheimer's and Parkinson's Diseases and Related Neurological Disorders (AD/PD ${ }^{\mathrm{TM}} 2017$ )

29 March-2 April 2017

Vienna, Austria

http://adpd2017.kenes.com/

Berlin BRAIN \& BRAIN PET 2017; 28th Symposium on Cerebral Blood Flow, Metabolism and Function \& 13th Conference on Quantification of Brain Function with PET

31 March-4 April 2017

Berlin, Germany

http://www.brain2017.net/

BNA2017 Festival of Neuroscience

10-13 April 2017

Birmingham, UK

https://www.bna.org.uk/events/view.php?permalink=QB7X4OQFWSs 